\title{
FILTER FAMILIES WITH MINIMUM TIME-BANDWIDTH PRODUCTS
}

\author{
Hrvoje Babic and Mladen Vucic \\ Faculty of Electrical Engineering and Computing \\ Unska 3, Zagreb, HR10000, Croatia
}

\begin{abstract}
A filter family with minimum product of the impulse response duration and the frequency bandwidth is considered. The impulse response spread is characterized by the higher order moments. For the frequency spread measure, the second order moment is used. Minimizing products of the moments, causal systems with the largest energy concentration in time for a given bandwidth are obtained. The resulting impulse response is quasi Gaussian with small and short ringing. The transfer function poles suitable for the filter design up to the eighth order are given.
\end{abstract}

\section{INTRODUCTION}

In many applications, the systems with small time spread of the impulse response for a given bandwidth are required. The real systems of the finite order typically have ringing. The general requirement is to make the ringing small and short. In the optimization procedure all these aspects should be present in the goal function. To have them all in an integral criterion, the use of higher order moments for characterization of the response spread is proposed. The moments have simple relations to the system function parameters. Therefore, the optimization can be carried out in the complex domain by varying zero-pole locations.

The frequency occupation of the band is determined by the signal shape and its time duration. To obtain the best shape realized as response of a finite order system, an optimization of the product

$$
\mathrm{P}_{\mathrm{nm}}=\alpha_{\mathrm{n}} \beta_{\mathrm{m}}
$$

of the time $\alpha_{\mathrm{n}}$ and the frequency $\beta_{\mathrm{m}}$ spread should be carried out.

For the spread $\alpha_{\mathrm{n}}$ and $\beta_{\mathrm{m}}$ various measures might be used [1]. The $n$-th central moment around delay $t_{d}$ of the squared impulse response $\mathrm{h}(\mathrm{t})$ normalized to the impulse response energy

$$
\alpha_{n}^{n}=\frac{\int_{-\infty}^{\infty}\left(t-t_{d}\right)^{n} h^{2}(t) d t}{\int_{-\infty}^{\infty} h^{2}(t) d t}
$$

has been used here for the time spread. The m-th moment of the squared amplitude response normalized to the energy

$$
\beta_{\mathrm{m}}^{\mathrm{m}}=\frac{\frac{1}{2 \pi} \int_{-\infty}^{\infty} \omega^{\mathrm{m}}|\mathrm{H}(\omega)|^{2} \mathrm{~d} \omega}{\frac{1}{2 \pi} \int_{-\infty}^{\infty}|\mathrm{H}(\omega)|^{2} \mathrm{~d} \omega} .
$$

has been used for the frequency spread measure.

As it is well known, the second order moments $n=2$ and $m=2$, have been used in the uncertainty principle for noncausal [1] and causal signals [2]. Here we use higher order moments $n=2,4,6$ and 8 for the impulse response characterization $\alpha_{n}$ and the second order moment $\beta_{2}$ for frequency response characterization. Motivation for such a decision is based on the fact that parabola $\left(\mathrm{t}-\mathrm{t}_{\mathrm{d}}\right)^{\mathrm{n}}$ is a weighting function in (2). In this way the contribution of the impulse response ringing in the spread measure $\alpha_{n}$ will be increased by $\mathrm{n}$. Minimizing the product (1) with such a measure, one can expect small and short ringing.

Systems with minimum spread of the impulse response given by higher order moments have already been analyzed [3]. There were no requirements given in the frequency domain. Here we use the product where the frequency domain is also taken into account. speed

The frequency response spread can be expressed by the second and higher order moments. The speed in which the frequency response of a real system approaches to zero is determined by number of poles and zeros. Therefore, higher order moments will not be able to modify significantly the frequency response form in the stopband. Thus, for the measure of the frequency response spread, the second moment will be sufficient. Also, the use of the second order moment has another important consequence. Namely, the integral (3) will converge for all system orders $\mathrm{N} \geq 2$. The integral (2) will converge regardless of used moment order.

\section{MOMENTS AND TRANSFER FUNCTIONS}

Time spread and bandwidth definitions (2) and (3), are suitable for causal functions too, only the integral limits have to be changed. Thus, we define a measure of impulse response spread by the $n$-th order central moment, and bandwidth as the second order moment, both normalized to the impulse response energy.

For optimization procedure in the complex domain, the criterion (1) should be expressed by the transfer function poles $p_{i}$, and zeros, $\mathrm{z}_{\mathrm{i}}$. The impulse response of the $\mathrm{N}$-th order filter with simple poles, and $\mathrm{M}<\mathrm{N}$ zeros, is given by

$$
h(t)=\sum_{r=1}^{N} K_{r} e^{p_{r} t}, \quad K_{r}=H_{0} \frac{\prod_{\substack{i=1 \\ j=1 \\ j \neq r}}^{M}\left(p_{r}-z_{i}\right)}{\prod_{\substack{j \\ j}}^{N}\left(p_{r}-p_{j}\right)},
$$


Table I. Transfer function parameters for $t_{d}=1$.

\begin{tabular}{|c|c|c|c|c|c|c|}
\hline & \multicolumn{3}{|c|}{$\mathbf{n}=\mathbf{2}$} & \multicolumn{3}{|c|}{$\mathbf{n}=\mathbf{4}$} \\
\hline $\mathbf{N}$ & $\omega_{\mathbf{p}}$ & $\mathbf{Q}_{\mathbf{p}}$ & $\omega_{\mathbf{3 d B}}$ & $\omega_{\mathbf{p}}$ & $\mathbf{Q}_{\mathbf{p}}$ & $\omega_{\mathbf{3 d B}}$ \\
\hline 2 & 1.4142 & 0.7071 & 1.4142 & 1.6175 & 0.6232 & 1.4037 \\
\hline 3 & 2.5543 & 0.9611 & 1.8233 & 2.6436 & 0.8086 & 1.8085 \\
& 1.4767 & & & 1.8062 & & \\
\hline \multirow{4}{*}{4} & 3.7816 & 1.2387 & 2.1697 & 3.7624 & 1.0123 & 2.1646 \\
& 2.1158 & 0.5914 & & 2.4139 & 0.5608 & \\
\hline \multirow{5}{*}{5} & 5.0551 & 1.5460 & & 4.9308 & 1.2279 & \\
& 3.0847 & 0.7702 & 2.5029 & 3.2975 & 0.6871 & 2.4922 \\
& 2.0967 & & & 2.5141 & & \\
\hline \multirow{6}{*}{6} & 6.3547 & 1.8903 & & 6.1264 & 1.4521 & \\
& 4.2073 & 0.9802 & 2.8122 & 4.3287 & 0.8383 & 2.8009 \\
& 2.6087 & 0.5626 & & 3.0179 & 0.5416 & \\
\hline \multirow{4}{*}{7} & 7.6694 & 2.2798 & & 7.3345 & 1.6802 & \\
& 5.4122 & 1.2100 & 3.0736 & 5.4404 & 0.9990 & 3.0806 \\
& 3.4795 & 0.7019 & & 3.8198 & 0.6362 & \\
& 2.5499 & & & 3.0887 & & \\
\hline \multirow{4}{*}{8} & 8.9926 & 2.7233 & & 8.5404 & 1.9014 & \\
& 6.6676 & 1.4586 & 3.3523 & 6.5946 & 1.1588 & 3.3486 \\
& 4.5385 & 0.8756 & & 4.7992 & 0.7521 & \\
& 2.9909 & 0.5491 & & 3.5567 & 0.5310 & \\
\hline
\end{tabular}

where $\mathrm{K}_{\mathrm{r}}, \mathrm{r}=1,2, \ldots, \mathrm{N}$ are the pole residues. Now, the $\mathrm{n}$-th order moment of the impulse response can be expressed as a function of poles and residues:

$$
\mathrm{m}_{\mathrm{n}}(\mathrm{h})=(-1)^{\mathrm{n}+1} \sum_{\mathrm{i}=1}^{\mathrm{N}} \sum_{\mathrm{j}=1}^{\mathrm{N}} \mathrm{K}_{\mathrm{i}} \mathrm{K}_{\mathrm{j}} \sum_{\mathrm{k}=0}^{\mathrm{n}} \frac{\mathrm{n} !}{\mathrm{k} !} \frac{\mathrm{t}_{\mathrm{d}}^{\mathrm{k}}}{\left(\mathrm{p}_{\mathrm{i}}+\mathrm{p}_{\mathrm{j}}\right)^{\mathrm{n}-\mathrm{k}+1}} .
$$

Impulse response energy can be obtained from (5) with $n=0$.

The second order moment of the frequency response can be expressed by the impulse response derivative, using Parseval's relation

$$
\frac{1}{2 \pi} \int_{-\infty}^{\infty} \omega^{2}|H(j \omega)|^{2} \mathrm{~d} \omega=\int_{-\infty}^{\infty}\left[\frac{\mathrm{dh}}{\mathrm{dt}}\right] 2 \mathrm{dt} .
$$

Expression (6) can also be computed from (5) using $\mathrm{K}_{\mathrm{r}} \mathrm{p}_{\mathrm{r}}$ that are residua of the impulse response derivative i. e.

$$
\mathrm{L}\left\{\frac{\mathrm{dh}}{\mathrm{dt}}\right\}=\mathrm{sH}(\mathrm{s}), \quad \frac{\mathrm{dh}}{\mathrm{dt}}=\sum_{\mathrm{r}=1}^{\mathrm{N}} \mathrm{K}_{\mathrm{r}} \mathrm{p}_{\mathrm{r}} \mathrm{e}^{\mathrm{p}_{\mathrm{r}} \mathrm{t}} .
$$

Thus, the second order moment of the amplitude response (6) is equal to zeroth moment of the impulse response derivative (7)

$$
\mathrm{m}_{0}\left(\frac{\mathrm{dh}}{\mathrm{dt}}\right)=-\sum_{\mathrm{i}=1}^{\mathrm{N}} \sum_{\mathrm{j}=1}^{\mathrm{N}} \frac{\mathrm{K}_{\mathrm{i}} \mathrm{p}_{\mathrm{i}} \mathrm{K}_{\mathrm{j}} \mathrm{p}_{\mathrm{j}}}{\mathrm{p}_{\mathrm{i}}+\mathrm{p}_{\mathrm{j}}}
$$

To ensure convergence of the moment integral (3), the number of zeros and poles should satisfy inequality

$$
2 \mathrm{M}+2 \leq 2 \mathrm{~N}-2 \text { or } \mathrm{N} \geq \mathrm{M}+2 .
$$

\begin{tabular}{|c|c|c|c|c|c|c|}
\hline & \multicolumn{3}{|c|}{$n=6$} & \multicolumn{3}{|c|}{$\mathrm{n}=\mathbf{8}$} \\
\hline $\mathbf{N}$ & $\omega_{p}$ & $Q_{p}$ & $\omega_{3 \mathrm{~dB}}$ & $\omega_{p}$ & $\mathbf{Q}_{p}$ & $\omega_{3 \mathrm{~dB}}$ \\
\hline 2 & 1.8564 & 0.5786 & 1.4635 & 2.1157 & 0.5535 & 1.5703 \\
\hline 3 & $\begin{array}{l}2.7983 \\
2.1237\end{array}$ & 0.7142 & 1.8099 & $\begin{array}{l}2.9944 \\
2.4364\end{array}$ & 0.6553 & 1.8472 \\
\hline 4 & $\begin{array}{l}3.8408 \\
2.7062\end{array}$ & $\begin{array}{l}0.8693 \\
0.5430\end{array}$ & 2.1370 & $\begin{array}{l}3.9752 \\
3.0022\end{array}$ & $\begin{array}{l}0.7767 \\
0.5318\end{array}$ & 2.1342 \\
\hline 5 & $\begin{array}{l}4.9427 \\
3.5220 \\
2.8782 \\
\end{array}$ & $\begin{array}{l}1.0355 \\
0.6376\end{array}$ & 2.4457 & $\begin{array}{l}.0227 \\
3.7666 \\
3.2258 \\
\end{array}$ & $\begin{array}{l}0.9091 \\
0.6048\end{array}$ & 2.4162 \\
\hline 6 & $\begin{array}{l}6.0805 \\
4.4812 \\
3.3655 \\
\end{array}$ & \begin{tabular}{|l|}
1.2093 \\
0.7557 \\
0.5305 \\
\end{tabular} & 2.7401 & $\begin{array}{l}6.1140 \\
4.6674 \\
3.7023 \\
\end{array}$ & \begin{tabular}{|l|}
1.0490 \\
0.6992 \\
0.5233 \\
\end{tabular} & 2.6906 \\
\hline 7 & $\begin{array}{l}7.2386 \\
5.5265 \\
4.1050 \\
3.4939 \\
\end{array}$ & $\begin{array}{l}1.3879 \\
0.8850 \\
0.6029\end{array}$ & 3.0171 & $\begin{array}{l}7.2341 \\
5.6579 \\
4.3937 \\
3.8716 \\
\end{array}$ & $\begin{array}{l}1.1946 \\
0.8056 \\
0.5806\end{array}$ & 2.9549 \\
\hline 8 & $\begin{array}{l}8.4041 \\
6.6225 \\
5.0115 \\
3.9398 \\
\end{array}$ & \begin{tabular}{|l|}
1.5669 \\
1.0182 \\
0.6962 \\
0.5235 \\
\end{tabular} & 3.2815 & $\begin{array}{l}8.3714 \\
6.7069 \\
5.2404 \\
4.2995 \\
\end{array}$ & $\begin{array}{l}.3437 \\
0.9186 \\
0.6575 \\
0.5185 \\
\end{array}$ & 3.2099 \\
\hline
\end{tabular}

\section{OPTIMIZATION PROCEDURE}

Pole and zero positions of causal filters with minimum timebandwidth product can be found by solving the problem
Table II. Transfer function parameters for $t_{d}=1$.

$$
\min _{z_{i}, p_{j}} P_{n 2}\left[z_{i}, p_{j}\right]
$$

We have carried out the optimization procedure for all-pole transfer functions. The frequency $\omega_{p}$ and quality factor $Q_{p}$ have been used, instead of the poles $\mathrm{p}_{\mathrm{j}}$. This variable set enables us to locate the pole pairs on the real axis, if necessary. Using $\omega_{\mathrm{p}}$ and $\mathrm{Q}_{\mathrm{p}}$, a rational transfer function can be written in the form

$$
H(s)=\frac{H_{0}}{\left(s+\omega_{p 0}\right) \prod_{j=1}^{(N-1) / 2}\left(s^{2}+\frac{\omega_{p j}}{Q_{p j}} s+\omega_{p j}^{2}\right)}
$$

for odd $\mathrm{N}$. When $\mathrm{N}$ is even, the linear factor in denominator is missing.

In a stable system $\omega_{p}$ and $Q_{p}$ are positive. Square values of goal function variables were applied to avoid constrained optimization procedure. Finally, optimum system poles and zeros were found as

$$
\min _{\omega_{\mathrm{p}}, \mathrm{Q}_{\mathrm{p}}} \mathrm{P}_{\mathrm{n} 2}\left[\omega_{\mathrm{p}}^{2}, \mathrm{Q}_{\mathrm{p}}^{2}\right]
$$

Optimization will force impulse response to concentrate around $t_{d}$ and practically extend to $2 t_{d}$. The parameter $t_{d}$ is chosen to be 1. This will not change the generality of the solution.

For searching minimum Quasi-Newton method with BFGS formula for Hessian matrix update [4] was used.

To get causal all pole filters with minimum time-bandwidth product, the optimization is carried out for moment orders $\mathrm{n}=2,4,6$ and 8 . 

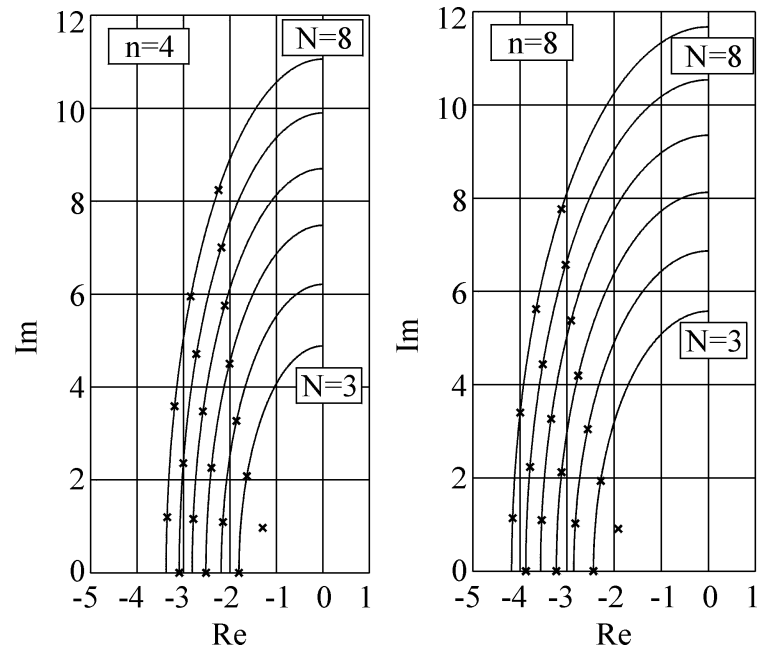

Figure 1. Pole positions of the optimum systems with $\mathrm{n}=4$ and $\mathrm{n}=8$, normalized to $\mathrm{t}_{\mathrm{d}}=1$.

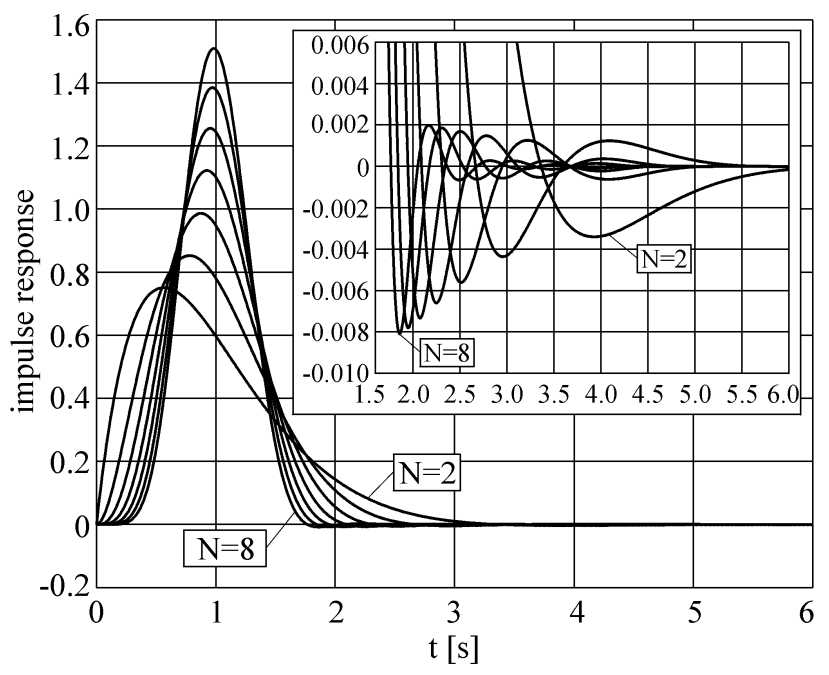

Figure 2. Impulse response of the optimum systems based on sixth moment, $t_{d}=1$.

\section{OPTIMIZATION RESULTS}

The numerical values of the pole parameters $\omega_{p}$ and $Q_{p}$, are given in Table I and Table II for systems from the second up to the eighth order.

For all-pole transfer functions with $t_{d}=1$, the examples of pole position are shown in Figure 1. It is interesting to note that poles are very nearly placed on ellipses with ellipses center located at the complex plane origin. The imaginary parts of poles are equidistant. Such properties are typical for linear phase and symmetric impulse response systems [5].

\subsection{The sixth moment system}

To illustrate behavior of the considered class of systems, the complete diagrams are given for the system with minimum product of sixth order moment in the time domain and second

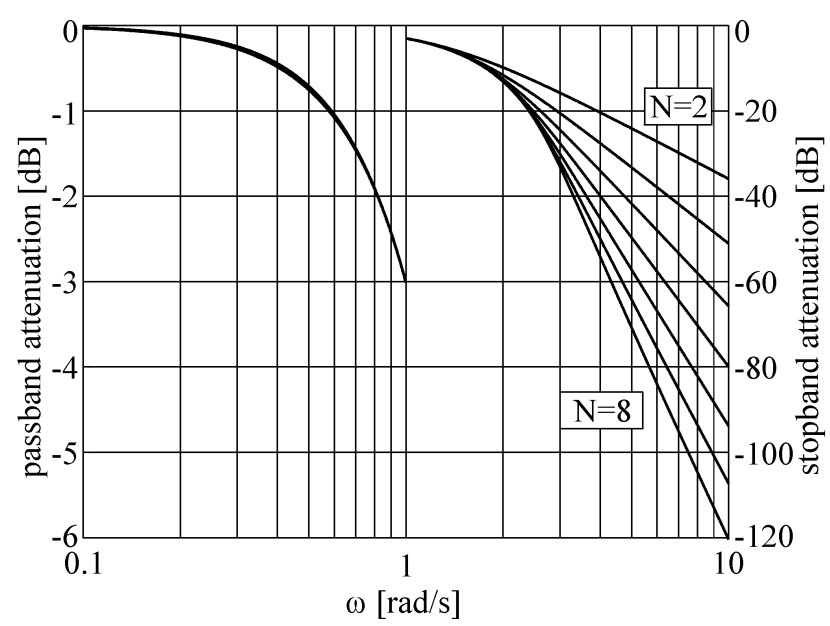

Figure 3. Amplitude response of the optimum systems based on sixth moment, $\omega_{3 \mathrm{~dB}}=1$.

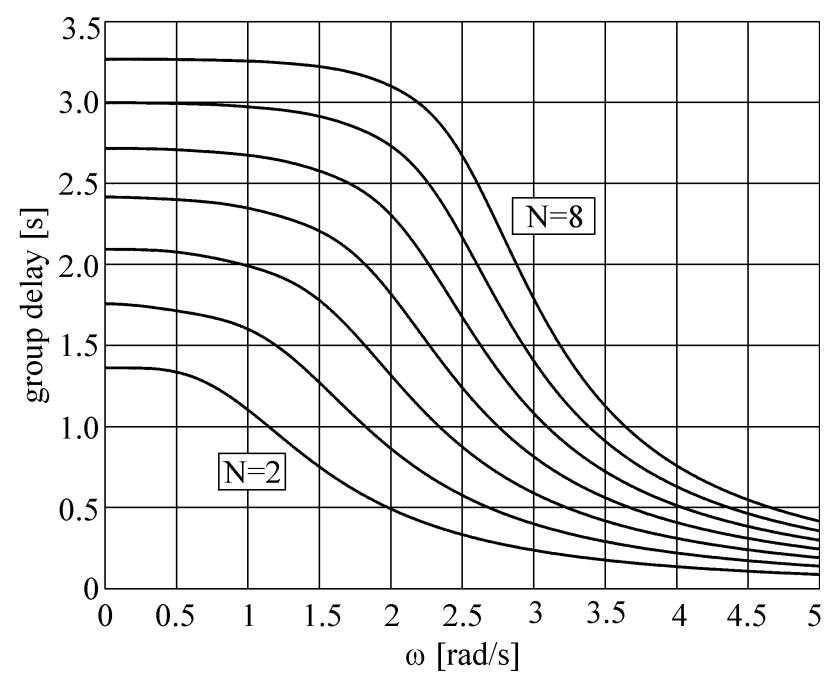

Figure 4. Group delay of the optimum systems based on sixth moment, $\omega_{3 \mathrm{~dB}}=1$.

order moment in the frequency domain. Impulse response is shown in Figure 2. It is a quasi Gaussian response, with small time spread. Undershoots are small $(<0.6 \%)$ and with short ringing. The step response overshoots are below $0.2 \%$ for $\mathrm{N} \geq 3$.

Amplitude and group delay responses are shown in Figure 3 and Figure 4, respectively, in a form suitable for comparison with classic filter approximations, given, for example, in [6]. The amplitude response is quasi Gaussian. The group delay curves illustrate an approximation of constant and they decrease monotonically as frequency decreases.

\subsection{Effects of moment order}

The optimization results for all moment orders are similar in character to the systems of sixth moment described above. The improvement in the impulse response i.e. shorter ringing has been obtained for the higher order moments, as it can be seen in 


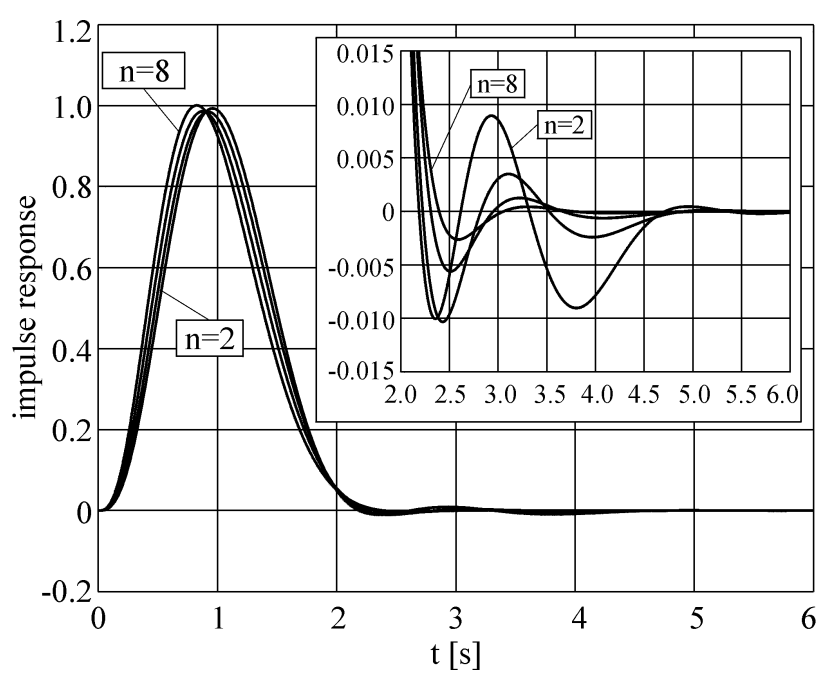

Figure 5. Impulse response of the optimum systems for various moment orders, $\mathrm{N}=4, \mathrm{t}_{\mathrm{d}}=1$.

Figure 5. The higher order moments with weighting function $\left(\mathrm{t}-\mathrm{t}_{\mathrm{d}}\right)^{\mathrm{n}}$ of higher power $\mathrm{n}$ apparently "punish" more the "tail" of the response. As a consequence, the ringing is shorter.

Undershoot of the impulse response is smaller than $2 \%$ for all considered moment orders and $\mathrm{N} \geq 3$, as shown in Figure 6. The data for Bessel filter are also given. The overshoot of the step response is smaller than $0.7 \%$ for all moment orders and $\mathrm{N} \geq 3$, Figure 7. Thus, the step response is very nearly monotonic.

The obtained product $\mathrm{P}_{\mathrm{n} 2}$ is a measure of the optimization performance. The conventional and more practical values for the time and frequency spread is, for example, the rise time $t_{r}$ $(10 \%-90 \%)$ and bandwidth $\omega_{3 \mathrm{~dB}}$ or $\mathrm{f}_{3 \mathrm{~dB}}$. The product is practically constant for $\mathrm{N}>3$ and it is $\mathrm{t}_{\mathrm{r}} \mathrm{f}_{3 \mathrm{~dB}}=0.347-0.342$.

Amplitude attenuation in stop band is higher for lower moments, and it is generally higher than at the Bessel filter [6]. Group delay approximate constants with curves which are getting more monotonic for higher order moments.

\section{CONCLUSION}

By the minimization of the time-bandwidth product using higher order moments, a new class of finite order systems has been obtained. Systems have the largest energy concentration in time for a given bandwidth. The impulse response has small and short ringing. Ringing duration is smaller for the higher system orders. Properties of obtained filter families can be favorable compared to similar filters with linear phase.

\section{ACKNOWLEDGEMENT}

This study was made at the Department for Electronic Systems and Information Processing of the Faculty of Electrical Engineering and Computing, University of Zagreb, Croatia. It was supported by Ministry of Science and Technology of Croatia, under grants No. 036024 and No. 036124.

\section{REFERENCES}

[1] Papoulis A. Signal analysis. McGraw-Hill Inc., 1977.

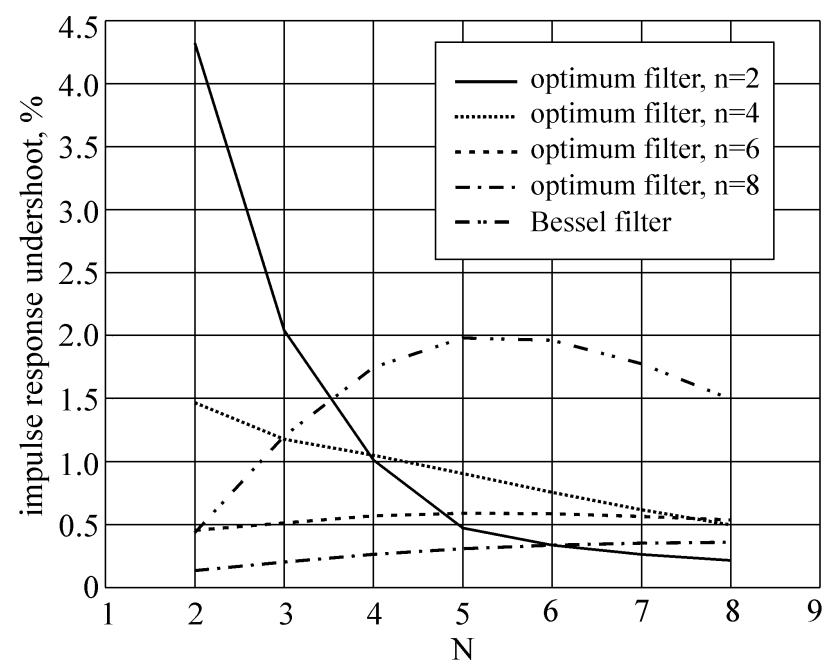

Figure 6. Impulse response undershoots of the systems for various moment orders and Bessel filters.

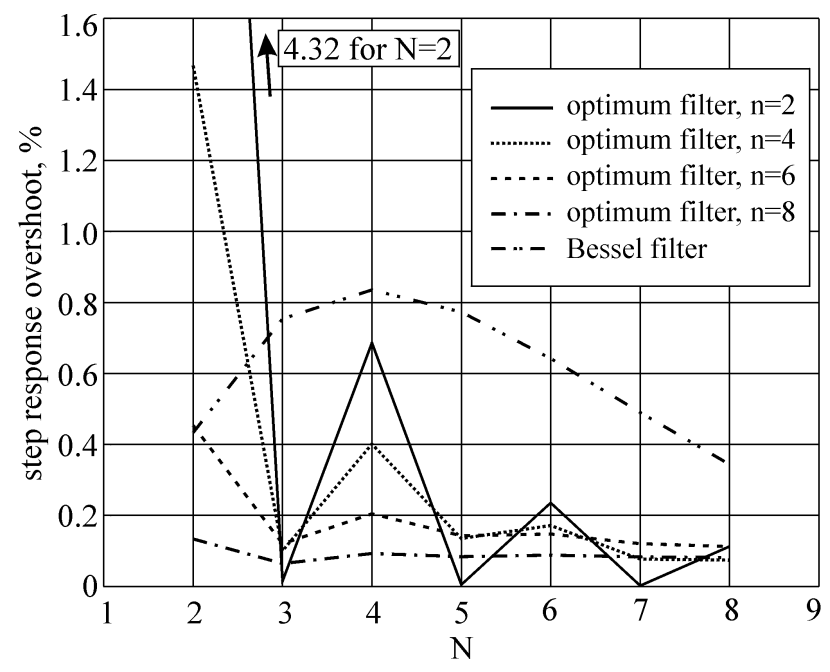

Figure 7. Step response overshoots of the systems for various moment orders and Bessel filters.

[2] Kuchenbecker H. P. Uber kausale Funktionen mit minimalem Zeit-Bandbreite-Produkt. Archiv der Elektrichen Ubertragung. H. 9, 1972, pages 259-264.

[3] Vucic M., Babic H., Filter Families with Minimum Impulse Response Moments. Proceedings of ISCAS 2000 - IEEE Symposium on Circuits and Systems. Geneva, Switzerland, 2000, pages 557-560.

[4] Fletcher R. Practical Methods of Optimization. Volume 1, John Wiley \& Sons, 1980.

[5] Vucic M., Babic H. "A Class of Systems with Symmetric Impulse Response". Proceedings of 1998 IEEE Symposium on Circuit and Systems. Volume 3, Monterey, CA, USA, 1998, pages 485-488.

[6] Zverev A. I. Handbook of Filter Synthesis. John Wiley \& Sons, 1967. 